\title{
Giovanni Bononcinis Oper Muzio Scevola (1710) und Johann Joseph Fux' Festa teatrale Costanza e Fortezza (1723). Vom musikalischen Drama zur Allegorie
}

\section{The Giovanni Bononcini's Opera Muzio Scevola (1710) and the Johann Joseph Fux' Festa teatrale Costanza e Fortezza (1723). From a Music Dramma to an Allegory}

Irena Veselá / irena.vesela@volny.cz

Moravian Library, Brno, CZ

\begin{abstract}
There are two operatic works with the same plot, the ancient story about the blocade of Rome by the Etruscan King Porsenna in the years 510-508 BC, compared in the article. Bononcini's opera Muzio Scevola (1710) was performed in Vienna at the occasion of the nameday of the Empress Amalia Wilhelmine. The true love and constancy of the women figures from Rome (Valeria and Elisa) are emphasised in this work. In the second work, however, the festa teatrale Costanza e Fortezza, which was performed in Prague in 1723 before the czech coronation of the imperial couple Charles VI. and Elisabeth Christine, is the same story presented as an allegory. The Romans defend their freedom with the help of their constancy and force against two enemies, who want to marry noble Roman girls Valeria and Clelia. By these means were shown the dynastic political intentions of Charles VI. and the momentousness of the pragmatic sanction.
\end{abstract}

\section{Keywords}

Costanza e Fortezza, Prague, 1723, Charles VI. (1685-1740), Elisabeth Christine von Braunschweig-Wolfenbüttel, Johann Joseph Fux, Pietro Pariati, Muzio Scevola, Giovanni Bononcini, Nicolò Minato, Silvio Stampiglia, Titus Livius, 18th century opera, festa teatrale 
Dieser Beitrag entstand im Rahmen der Untersuchung der musik-dramatischen Werken, die im Jahre 1723 anlässlich der Krönung Kaiser Karls VI. zum König von Böhmen in Prag aufgeführt wurden, besonders des zweifelsohne bedeutendsten Werkes, festa teatrale Costanza e Fortezza vom Hofkomponisten Johann Joseph Fux und Librettisten Pietro Pariati. Es wurde schon mehrmals festgestellt und aufgrund des Vergleichs mit zwei unmittelbar vorangegangenen Opern Teofane (Dresden 1719) und Adelaide (München 1722) zu Hochzeiten den beiden Töchtern des Kaisers Joseph I. bewiesen, dass die Handlung von diesem Huldigungswerk an die aktuellen dynastisch-politischen Botschaften angepasst wurde. ${ }^{1}$ In diesem Aufsatz wird Costanza e Fortezza mit einem dreizehn Jahre älteren Bühnenwerk Muzio Scevola von Giovanni Bononcini und Nicolò Minato, bzw. Silvio Stampiglia verglichen, in dem dasselbe Sujet bearbeitet worden war. Aufgrund des Vergleichs soll gezeigt werden, wie dieses Sujet von verschiedenen Librettisten unterschiedlich, dem Auftrag und der konkreten Gelegenheit nach, entweder als Vorlage für ein Musikdrama oder für ein Huldigungswerk behandelt werden kann und ob man durch die Unterschiede in der einzelnen Bearbeitungen die dynastisch-politische Absichten des Kaisers Karl VI. besser entdecken kann.

In den beiden am kaiserlichen Hof in Wien entstandenen Bühnenwerken, wurde die Geschichte über die tapfere und siegreiche Abwehr der Römer von der etruskischen Übermacht in den Jahren 510-508 vor Christi bearbeitet, die aus dem Werk Historiae $a b$ Urbe Condita von Titus Livius übernommen wurde. Der Entschluss der römischen Bürger wurde im ersten Rang durch die Taten der römischen Helden offensichtlich: Mutius Scevola, der ohne Erflog versuchte, den etruskischen König Porsenna zu töten, und danach legte vor den Augen Porsennas seine rechte Hand ins Feuer, um sie zu bestrafen, Horatius Cocles, der ganz allein die Brücke Ponte Sublicio vor dem Angriff der etruskischen Truppen abgewehrt hatte, und die römische Jungfrau Cloelia, die aus dem etruskischen Lager, wo sie als Geisel gehalten wurde, in der Nacht entflohen ist, wobei sie auf dem Pferd den Fluss Tiber durchschwommen habe.

Diese Geschichte wurde in der zweiten Hälfte des 17. Jahrhunderts als ein dramatischer Stoff mehrmals in Musik gesetzt. Schon im Jahre 1665 schuf der Dichter Nicolò Minato in Venedig ein Libretto Mutio Scevola, das von Komponisten Francesco Cavalli vertont worden war. ${ }^{2}$ Sein Libretto wurde dann bis dem Jahre 1700 für die auf verschiedenen, meistens italienischen Opernbühnen aufgeführten Werken verwendet. ${ }^{3}$ Im Jahre

1 Vgl. VÁCHA, Štěpán - VESELÁ, Irena - VLNAS, Vít - VOKÁČOVÁ, Petra. Karel VI. a Alžběta Kristýna. Česká korunovace 1723. Praha-Litomyšl : Paseka-Národní galerie, 2009. Eosdem. Karl VI. \& Elisabeth Christine. Die böhmische Krönung 1723. Frühneuzeit-Info, 21 (2010), S. 226-231. VESELÁ, Irena: „Venga quel Dì felice!“ Dynastisch-politische Botschaften der musikalischen Huldigungswerken für Karl VI. und Elisabeth Christine (1723). In TELESKO, Werner (hg.). Die Repräsentation der Habsburg-Lothringischen Dynastie in Musik, visuellen Medien und Architektur / Representing the Habsburg-Lorraine Dynasty in Music, Visual Media and Architecture 16181918. Wien-Köln-Weimar : Böhlau Verlag, 2017, S. 135-157. VESELÁ, Irena. „Costanza e Fortezza“ - Opera as a Political Manifesto. In The Performance of Diplomacy in Early Modern World (im Druck).

2 Vgl. DUBOWY, Norbert. Minato, Graf Nicolò. MGG Perosnenteil, Bd. 12, Kassel-Basel-London-New YorkPrag : Bärenreiter / Stuttgart-Weimar: Metzler, 2004, Sp. 243.

3 Vgl. SARTORI, Claudio. I libretti italiani a stampa dalle origini al 1800. Cuneo : Bertola \& Locatelli, 1993, Bd. IV. (L-Q), S. 203-204. Nach dem Jahre 1712 entstanden auch mehrere Werke mit dem Titel Porsena (Porsenna), in denen das gleichnamige Libretto von Agostino Piovene vertont worden war, siehe ebenda, S. 454. 
1695 wurde es vom Dichter Silvio Stampiglia überarbeitet und von mehreren Komponisten vertont; auch von Giovanni Bononcini, der damals in Rom mit Stampiglia zusammenarbeitete. ${ }^{4}$ Bononcini übersiedelte im Jahre 1697 zum Wiener Kaiserhof, wohin im Jahre 1706 auch Stampiglia kam. ${ }^{5}$ Dort wurde das Libretto unter dem Titel Muzio Scevola von Giovanni Bononcini vertont und am 30. Juni 1710 anlässlich des Namenstages der regierenden Kaiserin Amalia Wilhelmine von Braunschweig-Lüneburg aufgeführt. ${ }^{6}$

Am 28. August 1723 wurde in Prag anlässlich des 32. Geburtstags der regierenden Kaiserin Elisabeth Christine ein umfangreiches Huldigungswerk mit demselben Stoff aufgeführt. Dieses Werk war nach der persönlichen Devise Karls VI. Costanza e Fortezza benannt worden. ${ }^{7}$

Den ersten Impuls zum Vergleich den zwei Werken, Muzio Scevola (1710) und Costanza e Fortezza (1723), gab eine Aufzeichnung im Catalogo musicale, einen handschriftlichen Verzeichnis der in der ersten Hälfte des 18. Jahrhunderts beim Kaiserhof aufgeführten Bühnenwerken und Oratorien. In diesem Verzeichnis sind einige aus dem ersten Jahrzenten des 18. Jahrhundert stammende Bühnenwerke Giovanni Bononcinis und anderen Komponisten wie Marc' Antonio Ziani oder Antonio Caldara erst unter den später für Karl VI. geschaffenen Werken eingereiht, und zwar immer ohne direkte Jahresangabe. So befindet sich auch die die Oper Muzio Scevola von Bononcini unter den im Jahre 1722 entstandenen Werken. ${ }^{8}$ Man kann also voraussetzen, dass diese älteren Bühnenwerke noch in den 20. Jahren des 18. Jahrhundert behandelt wurden und dass Muzio Scevola eventuell als ein Muster zu Costanza e Fortezza dienen konnte.

Zuerst soll das Musikdrama Muzio Scevola in Betracht gezogen werden.

In dieser Oper treten sowie die beim Titus Livius erscheinende, als auch einige später zugefügte Figuren auf: Der etruskische König Porsenna, sein Schützling, der verbannte römische Thronfolger Tito Tarquinio und der von Librettisten Minato zugefügte

4 Vgl. LINDGREN, Lowell. Stampiglia, Silvio. Oxford music Online [cit. 2018-01-30]. URL: <http://www. oxfordmusiconline.com/subscriber/article/grove/music/26537?q=Muzio+Scevola\&search=quick\&pos=10\&_ start=1\#firsthit>

5 Ebenda.

6 HADAMOWSKY, Franz. Barocktheater am Wiener Kaiserhof. Mit einem Spielplan (1625-1740). Jahrbuch der Gesellschaft für Wiener Theaterforschung: Wien, 1951, S. 100. BONONCINI, Giovanni. Muzio Scevola: Drama per Musica Nel Giorno del gloriosissimo Nome Della S. C. R. M. Dell'Imperadrice Amalia Willelmina. Partitur, 3 Bd. A-Wn, Sign. Mus.Hs. 18269, URL: http://data.onb.ac.at/rec/AC14261168; Der MUZIO SCEVOLA. An dem Glorwürdigsten Nahmens-Tags Ihro Röm. Kayserl. Majestät Amalia Wilhelmine, Auff Allergnädigsten Befelch Ihrer Röm. Kayserl. vnd Königl. Majest. Josephi Deß Ersten wälsch-gesungener vorgestellt ... mit der Music zu denen Worten des Giovanni Bononcini. Wien: Cosmerovische Erben, 1710.

7 FUX, Johann Joseph. Costanza e fortezza : Festa teatrale per musica, da rappresentarsi nel reale castello di Praga. Per il ... giorno natalizio della sac. ces. catt. reale maesta di Elisabetta Cristina, imperadrice regnante, per commando della sac. ces. catt. reale maesta di Carlo VI., imperadore de' Romani. Partitur. 3 Bd. A-Wn, Sign. Mus.Hs. 17266 , http://data.onb.ac.at/rec/ AC14316057; PARIATI, Pietro. Costanza e fortezza, festa teatrale per musica da rappresentarsi nel reale castello di Praga per il ... giorno natalizio di Elisabetha Cristina, Imperatrice regnante ... Wien: J. P. van Ghelen, 1723; WELLESZ, Egon (ed.). Johann Joseph Fux. Costanza e Fortezza (=DTÖ, XVIII. Jhg.), Wien : Artaria, 1910.

8 CATALOGO Musicale. Continente Opera, Festa, Serenate, e Oratori Sacri. Handschrift. A-Wn, Sign. Mus. Hs.2453, Fol. 43r. Außer Muzio Scevola sind hier in ähnlicher Weise auch Bononcinis Opern Julio Ascanio Re d'Alba, Endimione, Turno Aricino und Abdolomino eingetragen. 
etruskische Feldherr Ismeno. Auf der römischen Seite steht der Konsul Publio Valerio Publicola, Muzio Scevola, Orazio Coclite und drei auch von Minato zugefügten römischen Frauen: Valeria, die Tochter des Konsuls Publio Valerio und Braut von Muzio Scevola, Elisa, die Gemahlin Orazios, und Vitellia, Tochter von Orazio und Elisa.

Am Anfang werden diese drei Frauen von den Etrusken verhaftet und als Kriegsbeute in den Feldlager Porsennas gebracht. Porsenna verliebt sich bald in Valeria. Sie lehnt seine Liebesneigung zwar sofort ab, sagt ihm jedoch nicht, dass sie Braut von Muzio ist. Elisa sagt wiederum ganz offen, dass sie treue Gemahlin Orazios ist und wehrt sich von Anfang an den Verlockungen Ismenos nachzugeben.

Orazio entscheidet sich zusammen mit Muzio ins Feldlager zu gehen und beide Römerinnen zu retten. Elisa schickt aber ihren Gemahl weg, damit sie, den Neigungen und Bedrohungen Ismenos widerstehend, selbst ihre Tugend und eheliche Treu bezeugen kann. Ismeno behandelt sowie sie, als auch ihre Tochter Vitellia übel und rücksichtlos und droht sogar Vittelia zu töten, um Elisa zur Liebe zu zwingen. Muzio begegnet im etruskischen Lager den Thronfolger Tarquinio und täuscht vor, die Römer verraten zu haben und ihm zum Regierungsantritt helfen zu wollen. Tarquinio glaubt ihm. Kurz danach versucht Muzio Porsenna zu töten, wird von den Etruskern verhaftet und bei nachfolgender Vernehmung legt er vor den Augen Porsennas seine rechte Hand ins Feuer. Porsenna verzeiht dem so tapferen Helden und neigt sich seitdem dem Friedenschluss mit Römer mehr als der Thronbesteigung Tarquinios zu. Er weiß nicht, dass Valeria und Muzio ein Liebespaar sind, und schickt Muzio als seinen Friedensboten nach Rom, damit er bei dem Konsul Publio Valerio die Hand Valerias für etruskischen König befragt. Muzio entscheidet sich das Wohl seiner Heimat vor seinem eigenen bevorzugen und auf Valeria verzichten. Publio Valerio stimmt der Heirat seiner Tochter mit Porsenna und den Friedenschluss zu. Erst zum Schluss wird die Liebe Valerias zu Muzio entdeckt und Porsenna verzichtet dann großzügig auf Valeria. Inzwischen entscheidet sich Elisa die Liebesneigung zu Ismeno vorzutäuschen. Sie verletzt ihn aber tödlich mit dem Schwert ihres Gemahls Orazio und flieht mit Vitellia aus dem Feldlager nach Rom, wo sich am Ende alle Figuren vereinigen. Ihre Tugend wird dann von allen bewundert. Zum Schluss erscheint die Göttin Flora, die den Namen der Kaiserin Amalia besingt.

In dieser für den Namenstag der Kaiserin bestimmten Oper wurden sehr stark die Standhaftigkeit und Treue der beiden verhafteten römischen Frauen hervorgehoben, besonders die Treue Elisas, die auch als ein Beispiel für ihre junge Tochter wirkt. Muzio Scevola kann für seine Tapferkeit und Bereitschaft, für das Wohl der Heimat auf seine Geliebte zu verzichten, geschätzt werden.

Im Jahre 1723 bearbeitete der italienische Hofdichter Kaiser Karls VI. Pietro Pariati die Geschichte für die festa teatrale Costanza e Fortezza zwar sehr ähnlich, hatte dabei doch ganz andere wichtige Momente akzentuiert.

Die Hauptfiguren (Porsenna, Tarquinio, Muzio, Orazio, Valeria und Publio Valerio) blieben im Libretto Pariatis erhalten. Konsuls Tochter Valeria tritt auch hier als die Liebespartnerin von Muzio auf. Dem Orazio gab Pariati statt Elisa als Liebespartnerin (nicht Ehefrau) die beim Livius erwähnte römische Jungfrau Clelia. Als die letzte Figur fügte er 
in die Handlung den Römer Erminio ein, einen Sohn des Konsuls Publio Valerio und Bruder Valerias. Damit wurde die fehlende Figur der jungen Römerin Vitellia ersetzt. Da es sich nicht um ein Musikdrama, sondern ein Huldigungswerk handelt, ließ Pariati alle zusätzlichen Figuren wie Feldherren, Minister, Herzfreunde der Hauptfiguren aus.

\begin{tabular}{|l|l|}
\hline \multicolumn{2}{|c|}{ Muzio Scevola, Wien $\mathbf{1 7 1 0}$} \\
\hline Die Römer & Die Etrusker \\
\hline Publio Valerio & \\
\hline Muzio Valeria & Porsenna \\
\hline Orazio Elisa & Ismeno \\
\hline Vitellia & Tarquinio \\
\hline
\end{tabular}

\begin{tabular}{|l|l|}
\hline \multicolumn{2}{|c|}{ Costanza e Fortezza, Prag $\mathbf{1 7 2 3}$} \\
\hline Die Römer & Die Etrusker \\
\hline Publio Valerio & \\
\hline Muzio Valeria & Porsenna \\
\hline Orazio Clelia & Tarquinio \\
\hline Erminio & \\
\hline
\end{tabular}

Tab. 1 Eine komparative Tabelle der Hauptfiguren in den beiden Libretti

Am Anfang befinden sich Valeria und ihr Bruder Erminio in der etruskischen Gefangenschaft. Porsenna droht die Stadt Rom zu zerstören und unmittelbar danach unternimmt er einen Angriff an Rom. Plötzlich taucht der Flussgott Tiber vom Fluss auf. Er warnt Porsenna und weist auf die römische Schutzgöttin Vesta hin, die gerade ihren Geburtstag feiert und die ihre Stadt vor der etruskischen Armee beschützt. Porsenna zieht sich zurück, versucht jedoch die Stadt mit anderen Mitteln zu gewinnen: er selbst liebt die gefangene Valeria und will sie Königin der Etrusker machen, Tarquinio will wiederum die edle römische Jungfrau Clelia heiraten und sie nach seinem Regierungsantritt auf den römischen Thron erheben. Unter diesen Bedingungen wollen Porsenna und Tarquinio das Frieden mit Rom schließen. Sowie beide Jungfrauen, als auch der römische Konsul Publio Valerio lehnen diese Heiratsanträge sofort ab.

Die starken und beständigen Römer beweisen durch ihre Heldentaten, dass sie ihrer neu gewonnenen Freiheit würdig sind und dass der listige und wortbrüchige Tarquinio dagegen gar nicht würdig ist, sie als König zu regieren. Porsenna erkennt diese Wahrheit schrittweise. Am Ende wird er zum Freund der Römer, mit denen er Frieden schließt und verzichtet gleichzeitig auf Valeria, währenddessen Tarquinio, mit dem Porsenna bisher viel Geduld hatte, von ihm verachtet wird. Seine Geduld reißt nachdem ihm Clelia mitteilt, dass Tarquinio ihre Unschuld bedroht habe und deswegen sie die gefährliche Flucht aus dem etruskischen Lager, wo sie sich als eine Geisel aufhaltete, nach Rom ergriff.

Durch ihre Stärke und Beständigkeit und unter dem Schutz von Göttin Vesta siegen die Römer über die Etrusker ohne Blut zu vergießen. Zum Schluss werden sowie 
diese Haupttugenden Kaisers Karl VI., die zwei tatsächlichen Sieger von dem römischetruskischen Konflikt, als auch die Göttin Vesta, die die Kaiserin Elisabeth Christine repräsentiert, gepriesen.

Setzt man zwei hier betrachtete Werke in Vergleich, kommt ans Licht, wie der Librettist Pariati mit der römischen Geschichte arbeitete, um ein an das konkrete Herrscherpaar gerichtetes Huldigungswerk zu schaffen und durch die Handlung die aktuelle dynastisch-politische Absichten Karls VI. erkennen zu lassen.

Die zwei Haupttugenden, Beständigkeit und Stärke, sind in den einzelnen Römer und Römerinnen zum Ausdruck gebracht, was am Ende auch offen gesprochen wird: Fan Costanza e Fortezza $i$ sommi Eroi. Da alle Römer unter ihren Einfluss handeln und alle in gleicher Weise sprechen müssen, scheinen sie, aus dem dramatischen Blick betrachtend, ein wenig unbelebt zu sein. Auch die dramatische Bearbeitung des ganzen Werkes wirkt, im Vergleich mit Muzio Scevola, ein bisschen statisch. Pietro Pariati konnte jedoch keine Römische Replik ins Gefahr einer doppelsinnigen Ausdeutung aussetzen und deshalb auch keinen der Römer schwanken zu lassen. Aus diesem Grunde verrät kein Römer die Republik und nicht einmal einen Verrat vortäuscht, wie Muzio in Muzio Scevola. Keine Römerin zeigt nicht einmal den Ansatz einer eventuellen, ob auch falschen, Neigung zu einem von den Feinden, wie das man bei Elisa in Muzio Scevola sieht. Die Römer bleiben immer stark, beständig und einig.

Der grundsätzliche Unterschied zwischen den beiden Libretti besteht jedoch in der Stellungsnahme auf den beiden Seiten im Verlauf der Friedensverhandlungen zu suchen. In Muzio Scevola wird der in Valeria verliebte etruskische König Porsenna von Tapferkeit und Beständigkeit Muzios berührt und deshalb will er den Konflikt mit der römischen Republik plötzlich beenden, ohne dem verbannten Tarquinio zum Thron geholfen zu haben: „Muzio, il tuo core inuitto / l'ardire eccelso, e la virtù Latina / più che le numerose aspre falangi / mi combatte, e mi vince. / Vivi, e libero torna, / che l'arsa man la tua costanza adorna. [...] Al Console di Roma / vattene, e di, che farò uscir le schiere / da i Romani confini / licentierò il Tarquini / lascierò $i$ Colle trionfato, e tutti / renderò $i$ prigionieri / pur che Valeria, che di Marte è preda / moglie in trofeo d'Amore / à me conceda. "9 Auf die Vorwürfe Tarquinios, der ihn für wortbrüchig, unbeständig und schwach haltet, antwortet Porsenna: „Di non ben giusta guerra / prouocator tu fosti, e se m'opponi / che m'induca alla pace il Dio d'Amore / anco à gloria m'arreco / che alla ragion m'apra le luci un cieco."10 Konsul

9 II. Akt 7. Auftritt, BONONCINI, Giovanni. Muzio Scevola, Bd. 2, Fol. 34v-35v (Siehe Anm. 5). - Mutzio / dein unüberwindliches Hertze / deine ruhmwürdige Khünheit / und die Römische Tugend hat mich mehrer als zahlreiche Kriegs-Schaaren befochten / und überwunden; Lebe / und khere gantz frey zuruck / Dann deine verbrennte Hande bezieret deine Beständigkeit [...] Gehe zu dem Römischen Burgermaister / sage ihme / das ich von denen Römischen Gräntzen mein Krieges-Hörr zuruck beruffen / den Tarquinio sambt seinen Anhang beurlauben / und den besigten Gianicolo sambt allen Gefangenen frey verlassen werde / wann er mir nur die Valeria / welche eine Beuthe deß Krieges ist / als ein SigesZeichen der Liebe zur Ehe will überlassen. Der MUZIO SCEVOLA, S. 33 (Siehe Anm. 6).

10 III. Akt, 10. Auftritt, BONONCINI, Giovanni. Muzio Scevola, Bd. 3, Fol. 57rv. - Du bist der Anfänger eines unbilligen Krieges gewesen / und wann du mir vorwerffest / daß mich der Liebes-Gott zu dem Friden verleuthe / so gerathet es mir zur Höchsten Ehre / daß meine durch deinem Ubermuth verfinsterte Augen von einem Blinden zur Billichkeit eröffnet werden. Der MUZIO SCEVOLA, S. 53 (Siehe Anm. 6). 
Publio Valerio freut sich über dem Friedensangebot Porsennas und sieht die eventuellen Konsequenzen der Eheverbindung seiner Tochter mit dem etruskischen König für die römische Republik und ihre Freiheit nicht über. Als ihm Muzio im zweiten Akt die Wille Porsennas mitteilt, antwortet er ohne Verzögerung: „Gran cose arrecchi, Roma / respirerà per te. Se può Valeria con Imenei felici l'afflitta Patria coronar d'oliuma / Facciasi. "11 Dass am Ende zur Eheschließung Valerias mit Porsenna nicht kommt, ist nicht durch die hartnäckige Bewahrung der Unabhängigkeit aus der Römischen Seite verursacht, sondern durch die Beständigkeit Muzios und durch die Liebe von Valeria und Muzio, der der großzügige etruskische König nachgibt: „Ah non sia vero / che di si nobil Alme / io disgiunga i legami, e quanto, ò Muzio / è nobile il tuo cor sia vile il mio. / Ti concedo Valeria. E sappia il mondo / che può in un Regio core / assai più la Virtù, che il Dio d'Amore. [...] Valeria, Muzio, Elisa, Orazio / ammiro i uostri pregi, e scorgo / che tutti son di Roma / degni d'eterni onori / le Donne, i Cavalier, l'armi e gli Amori."12

In Costanza e Fortezza wird der Friedens- und Heiratsantrag Porsennas und Tarquinios von allen Römern streng abgelehnt, da durch diese eventuellen Bände würde das Königtum mit dem tyrannischen Tarquinio in Rom wiedererrichtet, und gleichzeitig würden neue dynastische Beziehungen mit dem fremden Königen entstehen. Auf der römischen Seite wird offen gesprochen: „Rei doni, e vane offerte! Il nostro sangue / Servir non puote a propagar tiranni und Le Patrie leggi / Stan contra i voti tuoi. Figlie Romane / Dar non denno gli eredi a' Re stranieri." ${ }^{13}$ Das von Porsenna und Tarquinio eröffneten Verhandlungen um das Frieden und um die Hände der Römerinnen wird hier als tatsächlich zum maßgebenden Spiel um die römische Freiheit. Die Römer sind bereit lieber zu sterben als an sie zu verzichten, was auch der Konsul Publio Valerio dem König Porsenna mitteilt: „Pace si vuol: ma da la pace intate / Restin le nostre leggi. Al Re Porsenna, / Esse vietan Valeria. E' comun voto / L'eccidio, anzi che i Regi. Il giorno estremo / Di nostra libretà, lo sia di Roma. / Cosi risponde il Popolo, e'l Senato. / In tua man sta la pace, e sta la guerra."14

11 II. Akt, 12. Auftritt, BONONCINI, Giovanni. Muzio Scevola, Bd. 2, Fol. 65v-66r. - Du hast mir sehr grosse un wichtige Sachen vorgetragen. Rom wird sich durch dich erhollen / wann die Valeria durch das beglückte Ehe-Bande ihr betrangtes Vatterlande mit Oliven-Zweygen krönen kan; so seye Dies erlaubet. Der MUZIO SCEVOLA, S. 38 (Siehe Anm. 6).

12 III. Akt, 13. und 14. Auftritt, Ebenda, Bd. 3, Fol. 69rv und 71v. Nein / ich kan das Bande zweyer so edlen Seelen nicht entzweyen / und deiner edlen Großmüthigkeit / O Mutzio / nicht in dem mindestem nachgeben; Ich überlasse dir die Valeria / und die Welt dises wohl erwege / daß in einem Königlichem Hertzen mehrer die Tugend als die Liebe vermöge. [...] Valeria / Mutzio / Elisa / Oratio / ich bewundere euere Unvergleichliche Tugenden / und mueß es frey bekennen / daß in Rom das Frauen-Zimmer / die Cavallier/ die Waffen und die Liebe ewiger Lorber-Kräntze würdig seye. Der MUZIO SCEVOLA, S. 56 und 57 (Siehe Anm. 6).

13 PARIATI, Pietro. Costanza e fortezza, S. 8 und 42 (Siehe Anm. 8), Die erste Replik gehört Erminio (I. Akt, 4. Auftritt), die zweite Muzio (III. Akt, 5. Auftritt). Die deutsche Fassung von Anton Prokoff nach: Sträfliche Geschencke / und eitele Antragungen! unser Geblüt ist nicht zur Fortpflanzung der Tyrannen gweidmet. - Unsere Gesätze seynd deinem Wünschen entgegen. Die Töchter deren Römern können und därffen anderwertigen Königen keines wegs die Erben pflantzen. PARIATI, Pietro. Die Stärcke und Beständigkeit / Theatral-Fest / An dem Glorwürdigsten GeburtsTag Der Römisch-Kaiserlich- wie auch Königlich-Spanisch-Catholischen Majestät ELISABETHAE CHRISTINAE...Auf Dero Königlichen Schloß zu Prag Welsch gesungen vorgestellet. Im Jahr 1723. In das Teutsche / von dem Herrn Prokoff... übersetzet. Wien : J. P. van Ghelen, 1723, S. 7 und 40.

14 III. Akt, 10. Auftritt, PARIATI, Pietro. Costanza e fortezza, S. 48-49 (Siehe Anm. 8). - Den Frieden wünschet man: aber einen Frieden der unsere Gesätze nicht verletze. Porsennae / dem König versaget man die Valeriam / und die 
Der durch die römische Stärke und Beständigkeit überwindete Porsenna stimmt am Ende mit diesem Frieden zu: „La Costanza ne apprezzo, e la Fortezza, / Cui trionfi predisse il Patrio Fiume, / Apprezzo in lei la Dea, / Che me salvò da mortal rischio; e in lei / Temo il cielo impegnato a sua difesa [...]. ${ }^{15}$ Si: voglio pace. I'cedo / De gli Aruspici a $i$ detti, a' vostri Numi, / A la vostra virtude, e a la gran Dea, / Che felici vi vuol."16 Die Römer gewinnen also das Frieden nicht dank der Bewunderung ihrer Treue und Beständigkeit, die Porsenna zum Ausdruck bringen würde und dank denen entschiede er sich die Belagerung Roms zu beenden. Dank dem Götterschutz und den Tugenden sind die Römer nämlich stärker als der bewaffnete etruskische König - und er gibt nur zum Schluss diese Tatsache zu.

Dieser Unterschied kann man auch in der Teilnahme der Gottheiten auf der dramatischen Handlung in den beiden Werken spüren. In Muzio Scevola werden von den handelnden Figuren nur das Schicksal oder die Götter im allgemein angesprochen. Am Ende kommt die Göttin Flora der Kaiserin Amalia Wilhelmina zu huldigen. In Costanza $e$ Fortezza tritt dagegen der Flussgott Tiber offenbar auf der Bühne auf. Der Fluss selbst spielt hier eine sehr bedeutende Rolle als ein Symbol - Porsenna und Tarquinio schreiten ihn nie über, nicht einmal während der Friedensverhandlungen, die immer auf der etruskischen Seite des Flusses geführt werden. In Muzio Scevola kommt im Gegenteil zum Schluss Porsenna nach Rom um sich mit Valeria zu vermählen und um die gefangene Römer zurück zu kehren.

Die wichtigste Gottheit in Costanza e Fortezza ist eindeutig die verborgene Göttin Vesta. Sie wirkt nicht nur zugunsten der Römer, sondern rettet auch Porsenna aus der Todesgefahr, wenn Muzio versucht ihn zu töten. In der Licenza erscheint der römische Genius und auch die Götter Penaten. Der römische Genius entdeckt nicht nur die Identität von Vesta, sondern auch die von der Stadt Rom - tatsächlich wurden hier die Böhmische Länder und Prag gepriesen.

Die oben vorgelegte Betrachtung der zwei auf der ersten Blick sehr ähnlichen, tatsächlich aber doch unterschiedlichen Bearbeitungen von demselben Stoff lasset uns vermuten, dass der Verfasser von Costanza e Fortezza Pietro Pariati aus dem älteren Werk seinen Vorgänger Nicolò Minato und Silvio Stampiglia hervorgehen konnte; den politischen Absichten des Auftraggebers Karls VI. nach ließ er jedoch die Geschichte nicht mit einem, sondern mit zwei zunichtegemachten königlichen Hochzeiten abgeschlossen zu werden, damit es offensichtlich und klar würde, dass niemand durch die eventuelle künftige Heiraten mit den zwei Töchtern Karls VI. Maria Theresia und Maria Anna die

allgemeine Zusammenstimmung ist / ehe sich zum Untergang als Königen zu bequemen bereit. Der Tag / der unserer Freiheit der letzte seyn solte / müste auch Rom der letzte Tag seyn. Also antwort das Volck / also der Römische Rat / Der Frieden / oder Krieg / dir frey gestellet hat. Vgl. PARIATI, Pietro. Die Stärcke und Beständigkeit, S. 46 (Siehe Anm. 13)

15 III. Akt, 9. Auftritt, PARIATI, Pietro. Costanza e fortezza, S. 46 (Siehe Anm. 8). - Ich rühme die Beständigkeit / und ihre Starckmut / der die Tyber Triumphierungen vorgesagt. Verehre in derselben die Göttin / die mich von der tödlichen Gefahr befreyet / und beförchte den Himmel / der sich ihrer annimmet. Vgl. PARIATI, Pietro. Die Stärcke und Beständigkeit, S. 44 (Siehe Anm. 13).

16 III. Akt, 10. Auftritt, PARIATI, Pietro. Costanza e fortezza, S. 49 (siehe Anm. 8). - Ja / ich verlange den Frieden. Ich folge deren Weissagern Aussag / ergebe mich eueren Göttern / eurer Tugend / und der grossen Göttin / die euch beglücken will. Vgl. PARIATI, Pietro. Die Stärcke und Beständigkeit, S. 47 (Siehe Anm. 13) 
Länder der Böhmischen Krone erben wird. Angesichts der damaligen dynastisch-politischen Absichten des sächsischen Kurfürsten, der seine zwei kleinen Enkel als eventuelle Bräutigame angeboten hatte, wurde dieses Thema sehr aktuell. Mit den zwei in der Oper nicht realisierten Hochzeiten konnte man auch besser auf die am Anfang erwähnte kurfürstliche Hochzeitsopern Teofane und Adelaide reagieren, in denen die glücklichen Hochzeiten von den zwei vornehmsten Prinzessinnen gefeiert wurden. Der Sieg der Römer ohne Blut kann den gewünschten Sieg der Pragmatischen Sanktion vorstellen.

Was sich der Musik betrifft, unterscheiden sich die beiden Werken auf den ersten Blick ganz wesentlich: die Oper Muzio Scevola von Bononcini ist eine typische damalige solistische Oper, in welcher am meisten die Soloarien erscheinen, während die festa teatrale Costanza e Fortezza von Fux ein seltenes, im Freien aufgeführtes und deswegen groß ausgestattetes Werk vorstellt, wo außer Soloarien auch mehrere Chorauftritte eine bedeutende Rolle spielen. Die Instrumentalbesetzung der Arien ist jedoch in den beiden Werken ähnlich: ein Streicherensemble (vierstimmiges oder mit Violini unisoni) mit der Basso-Continuo-Begleitung. In Muzio Scevola ist beinahe die Hälfte von allen Soloarien nur mit den Basso-Continuo-Instrumenten begleitet; doch konnte sich Bononcini erlauben, in einigen Arien die Klangfarbe der solistischen Blechblas- oder Holzblasinstrumenten (Trompete, Chalumeau, Traverso, Fagott) einsetzen, um dadurch einen konkreten Affekt der handelnden Figur oder Stimmung zu betonen. Nur der Hauptfigur Muzio Scevola widmete der Komponist im ersten Akt eine Aria mit Trompete und im zweiten Akt eine Aria mit der Solostimme von Tiorba, die mit Violinen konzertiert. Fux konnte im Gegenteil keine konzertierenden Solostimmen verwenden; trotzdem kann man in der musikalischen Besetzung der Costanza e Fortezza eine Klangsymbolik finden. Sie beruht hier hauptsächlich im Trompetenschall: Am Anfang begleiten diese Instrumente die kampfsüchtigen Etrusker, während die Römer nur mit Basso-Continuo begleitet werden. Danach klingen die Trompeten am Ende des I. Aktes, vor und während dem Gefecht Orazios mit den Etruskern auf der Brücke Ponte Sublicio, der mit Rettung Orazios und Preisen der Vesta endet. Dann schweigen die Trompeten bis zum Schluss des Werkes, wo sie den Chorauftritt der siegreichen Römer begleiten. Die Etrusker müssen sich zum Schluss im Gegenteil nur mit Basso-Continuo-Begleitung begnügen.

Der Vergleich dieser zwei Bühnenwerke lässt uns beobachten, durch welchen, manchmal kaum bemerkbaren dramatischen Mitteln kann die altertümliche Geschichte entweder als ein Musikdrama oder als ein ganz sozusagen im Dienst der Staatsidee ${ }^{17}$ stehendes allegorisches Huldigungswerk bearbeitet werden.

The article was funded by Institutional support for long term conceptual development of research organization (Moravian Library) by Czech Ministry of Culture.

17 Vgl. MATSCHE, Franz: Die Kunst im Dienst der Staatsidee Kaiser Karls VI. Berlin-New York: Walter de Gruyter, 1981. 


\section{Bibliography}

\section{Sources}

A-Wn, Sign. Mus.Hs. 18269

BONONCINI, Giovanni. Muzio Scevola: Drama per Musica Nel Giorno del gloriosissimo Nome Della S. C. R. M. Dell'Imperadrice Amalia Willelmina. Partitur. 3 Bd. URL: <http://data.onb.ac.at/rec/ AC14261168>.

A-Wn, Sign. Mus.Hs.2453

CATAlOGO Musicale. Continente Opera, Festa, Serenate, e Oratori Sacri.

A-Wn, Sign. Mus.Hs. 17266

FUX, Johann Joseph. Costanza e fortezza : Festa teatrale per musica, da rappresentarsi nel reale castello di Praga. Per il ... giorno natalizio della sac. ces. catt. reale maesta di Elisabetta Cristina, imperadrice regnante, per commando della sac. ces. catt. reale maesta di Carlo VI., imperadore de' Romani. Handschriftl. Partitur. 3 Bd., URL: <http://data.onb.ac.at/rec/ AC14316057>.

Der MUZIO SCEVOLA. An dem Glorwürdigsten Nahmens-Tags Ihro Röm. Kayserl. Majestät Amalia Wilhelmince, Auff Allergnädigsten Befelch Ihrer Röm. Kayserl. vnd Königl. Majest. Josephi Deß Ersten wälsch-gesungener vorgestellt ... mit der Music zu denen Worten des Giovanni Bononcini. Wien : Cosmerovische Erben, 1710.

PARIATI, Pietro. Costanza e fortezza, festa teatrale per musica da rappresentarsi nel reale castello di Praga per il ... giorno natalizio di Elisabetha Cristina, Imperatrice regnante ... Wien : J. P. van Ghelen, 1723.

PARIATI, Pietro. Die Stärcke und Beständigkeit / Theatral-Fest / An dem Glorwürdigsten Geburts-Tag Der Römisch-Kaiserlich- wie auch Königlich-Spanisch-Catholischen Majestät ELISABETHAE CHRISTINAE...Auf Dero Königlichen Schloß zu Prag Welsch gesungen vorgestellet. Im Jahr 1723. In das Teutsche / von dem Herrn Prokoff...übersetzet. Wien : J. P. van Ghelen, 1723.

WELLESZ, Egon (ed.). Johann Joseph Fux. Costanza e Fortezza (=DTÖ, XVIII. Jhg.), Wien: Artaria, 1910.

\section{Literature}

DUBOWY, Norbert. Minato, Graf Nicolò. MGG Perosnenteil, Bd. 12, Kassel-Basel-London-New York-Prag: Bärenreiter / Stuttgart-Weimar: Metzler, 2004, Sp. 243.

HADAMOWSKY, Franz. Barocktheater am Wiener Kaiserhof. Mit einem Spielplan (1625-1740). Jahrbuch der Gesellschaft für Wiener Theaterforschung : Wien, 1951.

LINDGREN, Lowell. Stampiglia, Silvio. Oxford music Online [cit. 2018-01-30]. URL: <http://www. oxfordmusiconline.com/subscriber/article/grove/music/26537?q=Muzio+Scevola\&search=qu ick\&pos=10\&_start=1\#firsthit >

MATSCHE, Franz: Die Kunst im Dienst der Staatsidee Kaiser Karls VI. Berlin-New York : Walter de Gruyter, 1981.

SARTORI, Claudio. I libretti italiani a stampa dalle origini al 1800. Cuneo : Bertola \& Locatelli, 1993, Bd. IV.

VÁCHA, Štěpán - VESELÁ, Irena - VLNAS, Vít - VOKÁČOVÁ, Petra. Karel VI. a Alžběta Kristýna. Česká korunovace 1723. Praha-Litomyšl : Paseka-Národní galerie, 2009. 
Eosdem. Karl VI. \& Elisabeth Christine. Die böhmische Krönung 1723. Frühneuzeit-Info, 21 (2010), S. 226-231.

VESELÁ, Irena: „Venga quel Dì felice!“ Dynastisch-politische Botschaften der musikalischen Huldigungswerken für Karl VI. und Elisabeth Christine (1723). In TELESKO, Werner (hg.). Die Repräsentation der Habsburg-Lothringischen Dynastie in Musik, visuellen Medien und Architektur / Representing the Habsburg-Lorraine Dynasty in Music, Visual Media and Architecture 1618-1918. Wien-Köln-Weimar : Böhlau Verlag, 2017, S. 135-157.

VESELÁ, Irena. „Costanza e Fortezza“ - Opera as a Political Manifesto. In The Performance of Diplomacy in Early Modern World (in print). 
